wilderness experience to assist with student exposure to new hazards when commencing at a coastal regional hospital. The coast has several hazards which are specific to the area.

Aim: To provide students with first responder education for situations commonly encountered on the Central Coast.

Methods: Sessions on first responder training specific to coastal and remote locations included: first aid and surf safety with the Ocean Beach Surf-Lifesaving Club and anti-venom education with the Australian Reptile Park. Education was provided regarding the transition from academic to clinical medicine including support and workload management. A two-day workshop was held on the Central Coast. Pre- and post-workshop surveys were conducted with a combination of matrix questions, Likert response scales, and long answer questions. Ethics was obtained. Both quantitative and qualitative responses were analyzed.

Results: Excellent feedback regarding this program was received. All students reported an increase in knowledge in all three domains of critical medicine and evacuation issues, student health and workload management, and Central Coast community and environment. The areas of greatest knowledge in each of these domains were the management of surf incidents, signs and symptoms of PTSD, and Central Coast marine and ocean environment. A confidence increase was seen in responding effectively to an emergency, particularly, in response to improvisation in the field. All findings were statistically significant with all $\mathrm{P}$-values $<0.01$.

Discussion: The addition of BEACCHEs to the orientation of medical students at the Central Coast Medical School has demonstrated to be an effective program for allowing students to adjust more quickly to the new clinical environment. Following the success of this program, BEACCHEs is expected to become part of the new Junior Medical Officer orientation in 2019.

Prehosp Disaster Med 2019;34(Suppl. 1):s142-s143

doi:10.1017/S1049023X19003169

Investigation of the Relationship Between Disaster Experiences and Disaster Measures: Potential for Specific Measures Against Disasters

Ms Hazuki Shintaku, Professor Hiromi Kawasaki, Assisstant

Professor Satoko Yamasaki, Mr. Yoshibiro Murata,

Mr. Luoming Huang, Professor Masayuki Kakehashi

Hiroshima University, Hiroshima-City, Minami-Ku, Japan

Introduction: Japan is known worldwide as an earthquakeprone country, and large-scale landslide disasters have occurred frequently in recent years. Early preparation is essential for taking precise action in case of an emergency. People with disaster experience are often discussed in the importance of evacuation drills. However, most people have no disaster experience, so awareness of disaster countermeasures is desirable for non-experienced people.

Aim: To clarify the concerns of non-experienced people and consider how to strengthen disaster measures as an evacuation drill host or educator.

Methods: From February to March 2018, we enrolled teachers and parents whose children attend Hiroshima City Elementary School. Based on disaster experiences, we divided them into two groups, non-experienced and experienced, and a comparison of measures was performed between them. We used SPSS ver.22 and did a chi-square test.

Results: There were 1,702 valid responses (145 teachers and 1,557 parents); 1,406 were non-experienced, and 289 were experienced. The issues both groups were most concerned about were "children's safety at school" (non-experienced 61.7\%, experienced 57.3\%), "securing food and drink at school" (39.0\%, 3.3.9\%), "acceptance and distribution of relief supplies" (28.1\%, 2.6.6\%), and "resident evacuation" (25.4\%, 2.4.0\%). The experienced were most concerned with "children's mental care" (60.2\%), and the non-experienced were most concerned with "children's safety at school" (61.7\%).

Discussion: Regardless of experience, parents tend to be deeply concerned about all things pertaining to their children. Physical safety, as well as psychological needs, were of high importance. For non-experienced, we should develop interest by focusing on children's needs when writing manuals for disaster measures and evacuation drills. Therefore, future projects to strengthen awareness of disaster prevention for the nonexperienced should focus on three key issues: "step-by-step approaches for children," "physiological needs," and "safety of schools and shelters."

Prehosp Disaster Med 2019;34(Suppl. 1):s143

doi:10.1017/S1049023X19003170

\section{Investigation on Level of First Aid Knowledge among Undergraduates}

Mr. Lin Run ${ }^{1}$, Ms Wang AoYu ${ }^{1}$, Ms Chen Yaqi ${ }^{1}$,

Mr. Lai Enjiang ${ }^{1}$, Dr. Hu Hai ${ }^{2,3,4}$

1. West China Medical School of Sichuan University, Chengdu, China

2. Emergency Office of West China Hospital, Sichuan University, Chengdu, China

3. China International Emergency Medical Team (Sichuan), Chengdu, China

4. Chinese National Comprehensive Emergency Medical Rescue Base, Chengdu, China

Introduction: First aid in a short time is the key to saving lives. As undergraduates step into society, they should have enough ability to save others. As a result, first aid knowledge and training are essential for them.

Aim: To investigate the cognition level of the first aid knowledge among undergraduates, and to improve the training for undergraduates.

Methods: A questionnaire was designed for this study. It includes the basic information of the test subjects, attitude towards first aid, level of first aid knowledge, etc. The subjects of the questionnaire were mainly undergraduates in Sichuan province.

Results: There were 302 valid questionnaires. The percent correct of the first aid basic knowledge quiz was only $47.62 \%$. Using Chi-Square tests to analyze, medical education can make a significant difference in the level of first aid knowledge. (Chi-Square $=251.004, \mathrm{P}<0.01$ ) Additionally, $78.81 \%$ of undergraduates thought it was significant to learn and master first aid knowledge, and the most common way to learn first aid knowledge was through university (81.46\%). 
Discussion: Universities should strengthen the training for undergraduates to improve their first aid skills. This is a feasible approach to promote a public level of first aid knowledge.

Prehosp Disaster Med 2019;34(Suppl. 1):s143-s144

doi:10.1017/S1049023X19003182

Issues of Which Organization is Responsible for Hospital Evacuation in Nuclear Disasters

Dr. Masaru Ogasawara ${ }^{1}$, Dr. Yuta Sato ${ }^{1}$, Dr. Katsunori Ito ${ }^{1}$, Dr. Kyoji Saito ${ }^{1}$, Dr. Katsubiro Ito ${ }^{2}$, Prof. Hiroyuki Hanada ${ }^{2}$

1. Aomori Prefectural Central Hospital, Aomori-shi, Japan

2. Hirosaki University, Hirosaki-shi, Japan

Introduction: At the time of a nuclear disaster, residents should evacuate from areas with high air dose rate. In the Great East Japan Earthquake, about $10 \%$ of patients died in a hospital evacuation in which medical teams were not involved in transportation.

Aim: To determine if hospital evacuation improved after the Fukushima nuclear accident.

Methods: This research investigates how the medical system of a nuclear disaster in Japan changed.

Results: There are 41 hospitals designated as Nuclear Emergency Core Hospitals, and they have 53 Nuclear Emergency Medical Assistance Teams (NEMAT; disaster medical dispatching team specialized in nuclear disasters consisting of medical doctors, nurses, and radiological technologists) that can support hospitals and information in the acute phase.

Discussion: At the time of a nuclear disaster, NEMAT is supposed to evacuate residents from the Urgent Protective Action Planning Zone (UPZ; within about $30 \mathrm{~km}$ radius). Tens of thousands to one million people live in this area. Hospital evacuation of more than several thousand patients is necessary. The entry of workers for transportation vehicles and lifeline restoration is limited within UPZ, so staying in a hospital is virtually impossible. There are over 2000 Disaster Medical Assistance Teams (DMAT), and many Red Cross Relief Teams; both of which are stipulated not to conduct clinical treatment in high dose areas and are not educated on nuclear disasters. Although there are Radiation Emergency Medical Assistance Teams (REMAT) consisting of doctors and technicians specializing in radiation medicine, they are few in number. They can perform dose assessment, but general medical care cannot be performed because an emergency physician is not included. Therefore, although NEMATs will conduct emergency and hospital evacuation in the affected area, the number of teams is too small to respond. The issue of which organization is responsible for massive hospital evacuation remains unsolved.

Prehosp Disaster Med 2019;34(Suppl. 1):s144

doi:10.1017/S1049023X19003194

\author{
The Knowledge, Attitudes, and Practices of Search and \\ Rescue Teams of Sri Lanka Army Regarding Search and \\ Rescue as a Response to Disasters \\ Dr. Saminda Kumara, Dr. Saveen Semage \\ Sri Lanka Army Health Services, Gampaha, Sri Lanka
}

Introduction: Sri Lanka Army is a valuable resource for the country as a capacity factor in disaster situations. Sri Lanka Army has established Search and Rescue teams (SAR teams) in all 25 districts.

Aim: To describe the knowledge, attitudes, and practices of SAR teams regarding search and rescue as a response to disasters.

Methods: A descriptive cross-sectional study was carried out from October to December 2017. Five platoons were selected randomly from high and medium risk district categories, and all five platoons were selected from the low-risk category. The total sample size was 465 . A pre-tested self-administered questionnaire was employed.

Results: The median age was 28 years. $3.2 \%$ were officers, $96.8 \%$ were soldiers, the majority $(80.4 \%)$ were educated up to G.C.E. (O/L), and $62.4 \%$ were married. $52.9 \%$ of the population had undergone SAR training during the past three years in Sri Lanka, and none had undergone training outside of the country. Overall knowledge regarding search and rescue as a response to disasters seem to be good (57.2\% received higher than a score of $75 \%$ ). $93.8 \%$ has desirable positive attitudes. $73.5 \%$ had participated in search and rescue operation as a response to disasters. Overall practices seemed to be poor, (71.3\% of the population received lower than a score of 75\%). A statistically significant association was observed with a level of education $(p=0.001)$, designation $(p=0.004)$, and knowledge on search and rescue as a response to disasters. Level of education, designation, and SAR training had no significant association with attitudes on search and rescue as a response to disasters. A statistically significant association was observed with designation $(p=0.021)$ and practices.

Discussion: Search and rescue drills should be carried out regularly. Knowledge of search and rescue as a response to disasters should be incorporated into training programs for officers and soldiers.

Prehosp Disaster Med 2019;34(Suppl. 1):s144

doi:10.1017/S1049023X19003200

Lack of Prioritization Causes Extended Time to Assessment of Severely Injured Trauma Patients in a Resource-Scarce Emergency Department

Dr. Maria Lampi ${ }^{1}$, MD John Tabu ${ }^{2}$, Dr. Johan Junker ${ }^{1}$, Professor Andreas Wladis $^{1}$

1. Centre for Teaching and Research in Disaster Medicine \& Traumatology, Linkoping, Sweden

2. Moi University College of Health and Science, Department of Disaster Risk Management, Eldoret, Kenya

Introduction: The time between injury and medical intervention is crucial in trauma care. Triage is essential to ensure prioritization and timely assessment of injured patients.

Aim: To investigate how the lack of triage system impacts timely intervention in a sub-Saharan hospital emergency department, and to investigate potential benefits of triage towards efficient management of trauma patients.

Methods: A prospective study including adult trauma patients admitted to the emergency department at Moi Teaching and Referral Hospital in Eldoret, Kenya, was conducted. Mode 